\title{
Efficacious Evaluation of Aloe Vera Tooth Gel and Commercially Available Tooth Gel on Patients with Gingivitis
}

\author{
Kanika $\mathrm{M}^{*}$ \\ Department of Periodontology and Oral Implantology, Laxmi Bai Dental College and Hospital, Patiala, Punjab, India
}

*Corresponding author: Kanika M, Department of Periodontology and Oral Implantology, Laxmi Bai Dental College and Hospital, Patiala, Punjab, India, Tel: 9888873083, E-mail: mohindrakanika@gmail.com

Citation: Kanika M (2018) Efficacious Evaluation of Aloe Vera Tooth Gel and Commercially Available Tooth Gel on Patients with Gingivitis. J Oral Health Dent Sci 2: 203

Article history: Received: 03 April 2018, Accepted: 16 May 2018, Published: 18 May 2018

\section{Abstract}

Objective: The purpose of the present study was to evaluate the efficacy of aloe vera tooth gel and commercially available tooth gel on patients with gingivitis.

Materials and Methods: This study was carried on 18 subjects who were randomly selected from individuals referred to Department of Periodontology and Oral Implantology, National Dental College and Hospital Derabassi. The study was based on the effect of aloe vera and close up tooth gel on gingiva. In this study subjects had undergone two sessions of oral hygiene practices in cross over manner. Clinical evaluation was undertaken using gingival index of Loe and Silness (1963) and plaque was assessed using Turesky, et al. modification of Quigley Hein Index (1970).

Results: Aloe vera containing tooth paste showed significant improvement in gingival and plaque index scores and results were comparable with close up containing tooth gel.

Conclusion: Aloe vera containing tooth paste may be a useful herbal formulation for plaque control agent and improvement in plaque and gingival status.

Keywords: Gingivitis; Anti-inflammatory; Anti- plaque

\section{Introduction}

Gingivitis and periodontitis are the two most common conditions affecting periodontium [1]. Dental plaque has been considered as a prime etiological factor whose presence lead to continuous disease activity. Oral hygiene failure leads to formation of pathogenic plaque which gives rise to gingivitis and if remain untreated may further give rise to periodontitis [2].

The first step involved in the treatment and prevention of gingival and periodontal disease is an effective plaque control. The best way to remove the harmful plaque from teeth and gums is to brush the teeth properly and regularly. Carefully performed daily plaque control has been demonstrated to reduce supragingival plaque and prevent disease progression. Tooth brush along with dentifrice is a useful aid for delivering therapeutic agents to teeth and gingiva and is most widely practiced form of oral hygiene in most of the countries. Dentifrices are available in the form of paste, gels, and powders [2].

The success of any toothpaste lies on its ability to eliminate pathogenic oral microflora.

Because of increasing awareness among people regarding the benefits of natural products e.g. neem, aloe vera, turmeric, the use of synthetic products is gradually declining. So the use of natural products in the prevention and treatment of oral conditions has increased. Among the various currently available herbal agents, Aloe vera (popularly known as "babosa") has been found to be beneficial. The gel or mucilage from Aloe barbadensis Miller (otherwise known as aloe vera) is a convenient homegrown remedy that can be used both as a moisturizing agent and for treating minor burns and skin abrasions. Aloe vera is a cactus like plant that actually is a part of the lily family. There are more than 300 varieties of the aloe plant but the Aloe barbadensis variety exhibits the best medicinal properties [3].

Its foliage, extract and resin, present antimicrobial, anti-inflammatory and healing properties. The dental uses of aloe vera are multiple; it is extremely helpful in treatment of gingivitis as it reduces bleeding of gums and acts as a powerful antiseptic in the periodontal pocket [4]. 
Modern use of aloe vera was first documented in the 1930s to heal radiation burns [3]. Aloe vera juice taken internally has been shown to have various beneficial effects on the body like irritable bowel syndrome and as a strong detoxifying agent [5].

The efficacy of Aloe barbadensis Miller increases when the plant is harvested after three years of growth but its nutritive potency decreases after 12 years of growth [6]. Aloe gel will lose its complete potency if it is exposed to sunlight for more than two hours, as it is easily oxidized; consequently, it is necessary to stabilize it under pharmaceutical standards for ready use and longer shelf life [6].

It is available in various forms like toothpaste (Forever Bright tooth gel), mouthwash (Optima Aloedent aloe vera mouth wash), as gel for healing in burns, stings, insect bites and many skin lesions, as topical spray- used for throat infections, painful teeth eruptions and joint pains.

Close Up tooth gel has a unique formula with active clean mouth wash that reaches all over the mouth and help to remove plaque.

The aim of this study is to compare the effect of alovera tooth gel and close up gel on plaque removal and gingival health.

\section{Materials and Method}

In the present study 18 subjects were selected from those visiting the Outpatient Department of Periodontology and Oral Implantology, National Dental College and Hospital, Derabassi (Punjab). All randomly screened participants were informed about the nature of the study.

The study went for a period of two months and was designed as randomized crossover study.

The subjects selected for the study had at least 20 natural teeth, with no supragingival calculus and other plaque retentive factors and with good manual dexterity and patient who showed willingness to participate in study. Subjects with medical disorders or probing depth $\geq 3 \mathrm{~mm}$, individuals under antimicrobial therapy at least 1 month prior to study and using mouthrinse and dentifrices containing substances containing with anti-inflammatory properties, as well as smokers and pregnant woman were excluded from the trial.

The participants were assigned to use both the test tooth gel (aloe vera) and controlled tooth gel (close up) and had to undergone two sessions of oral hygiene practices in a crossover manner.

One week before the start of the study, participants received an intraoral examination and oral prophylactic treatment. The participants were then instructed to continue their oral hygiene routine for a week. One week later participants underwent baseline registration of gingival inflammation and plaque deposits. Subjects were provided either with the close-up tooth gel or aloe vera tooth gel for the following three weeks. Patients had to brush their teeth twice daily in the morning and in the evening, and to refrain from other oral hygiene aids during the period.

After three weeks, participants again underwent registration of gingival inflammation and plaque deposits and it was followed by professional tooth cleaning. The participants were then instructed to continue their routine oral hygiene practices for the following week (Washout period) to avoid the carry over effect of the previously used material.

One week later, again registration of gingival inflammation and plaque deposits were recorded. Now, the cross-over of the material was done and participants were instructed to use either close up or aloe vera tooth gel for the following three weeks. After three weeks later, recording of gingival inflammation and plaque deposits was done.

\section{Clinical examination}

Clinical examination comprised of gingival index and plaque index. Plaque was stained using erythrosine solution and scored according to Turesky Quigley Hein Plaque index (1970). The status of gingival health was assessed by Loe and Silness (1963) for selected teeth.

\section{Statistical analysis}

Mean and standard deviation of plaque and gingival scores were calculated. Further statistical analysis was done by paired and unpaired t- test. In each group, the mean scores of gingival and plaque index was compared between baseline and end of the trial by Wilcoxon test. Results were presented as mean and standard deviation.

\section{Results}

The test dentifrice had a good acceptance and did not show adverse effects, such as formation of abscess and ulcerations or allergic reactions. 18 patients completed the two months study period, out of which 9 were assigned to test group and 9 to control group in a crossover manner.

Table 1 shows the overall means and standard deviation for both aloe-vera and close-up at base line and after 3 weeks of use.

The mean value of Quigley Hein index of aloe-vera at baseline and after 3 weeks of use is $1.3022 \pm .28275$ and $1.2167 \pm .25029$ and the 
p value came out to be 0.025 (Table 1). This shows that there was significant reduction in plaque levels. The mean value of Quigley Hein index of close up at baseline and after 3 weeks of use are $1.2650 \pm .13691$ and $1.2144 \pm 0.12789$ and $p$ value came out to be 0.001 . There was significant reduction in plaque levels.

\begin{tabular}{|c|c|c|c|c|c|}
\hline & & $\begin{array}{l}\text { QUIGLEY HEIN } \\
\text { INDEX } \\
\text { Mean } \pm \text { SD }\end{array}$ & $p$ value & $\begin{array}{l}\text { GINGIVAL } \\
\text { INDEX } \\
\text { Mean } \pm \text { SD }\end{array}$ & p value \\
\hline \multirow[b]{2}{*}{ ALOE VERA } & BASELINE & $1.3022 \pm .28275$ & \multirow[b]{2}{*}{0.025} & $1.3233 \pm .12993$ & \multirow[b]{2}{*}{0.096} \\
\hline & $\begin{array}{c}\text { AFTER } 3 \\
\text { WEEKS USE }\end{array}$ & $1.2167 \pm .25029$ & & $1.2800 \pm .11376$ & \\
\hline \multirow[b]{2}{*}{ CLOSE-UP } & BASELINE & $1.2650 \pm .13691$ & \multirow[b]{2}{*}{0.001} & $1.2522 \pm .10074$ & \multirow[b]{2}{*}{0.93} \\
\hline & $\begin{array}{c}\text { AFTER } 3 \\
\text { WEEKS USE }\end{array}$ & $1.2144 \pm .12789$ & & $1.2320 \pm .19245$ & \\
\hline
\end{tabular}

Table 1: Comparative analysis of mean values of Quigley hein index and gingival index scores for aloe vera and close-up group

With regard to gingival index there was no significant reduction both by aloe vera and close up with the baseline value for aloe vera

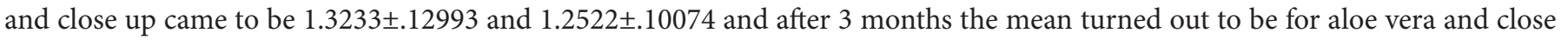
up $1.2800 \pm 0.11376$ and $1.2320 \pm .1924$ (Figure 1 and 2).

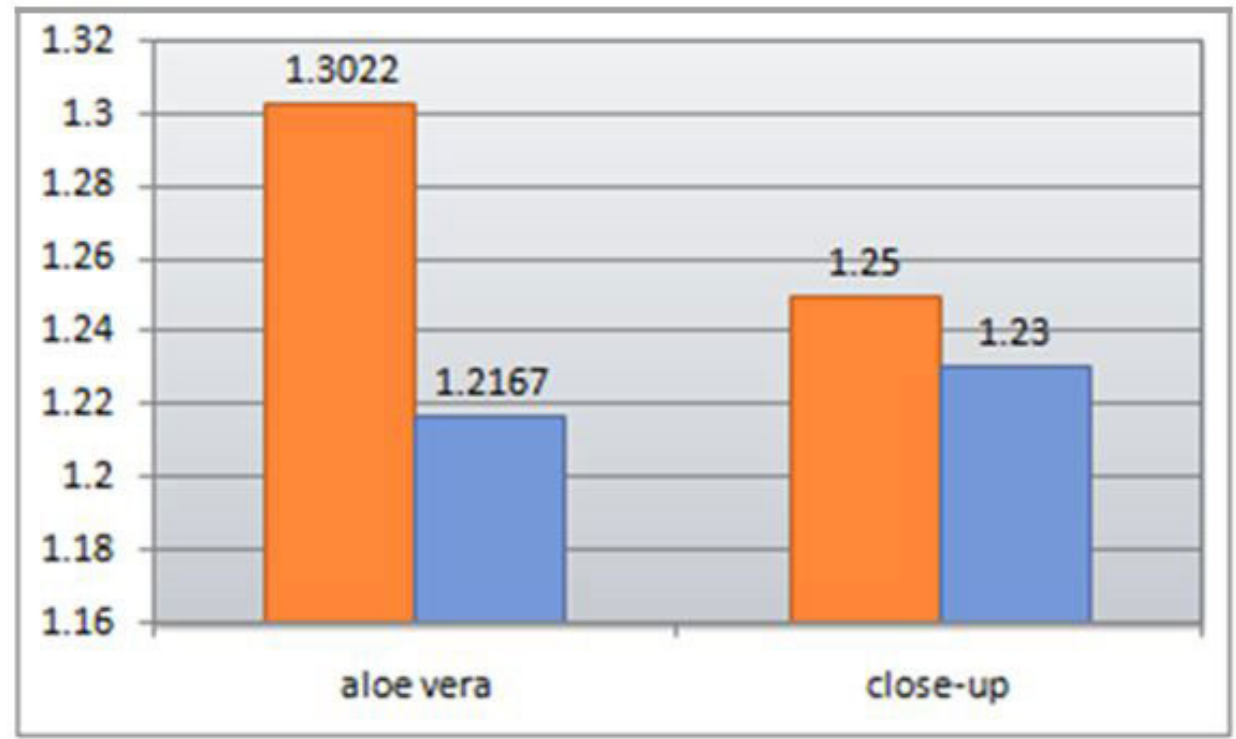

Figure 1: Bar diagram showing the mean Quigley hein index scores for aloe vera and close-up group

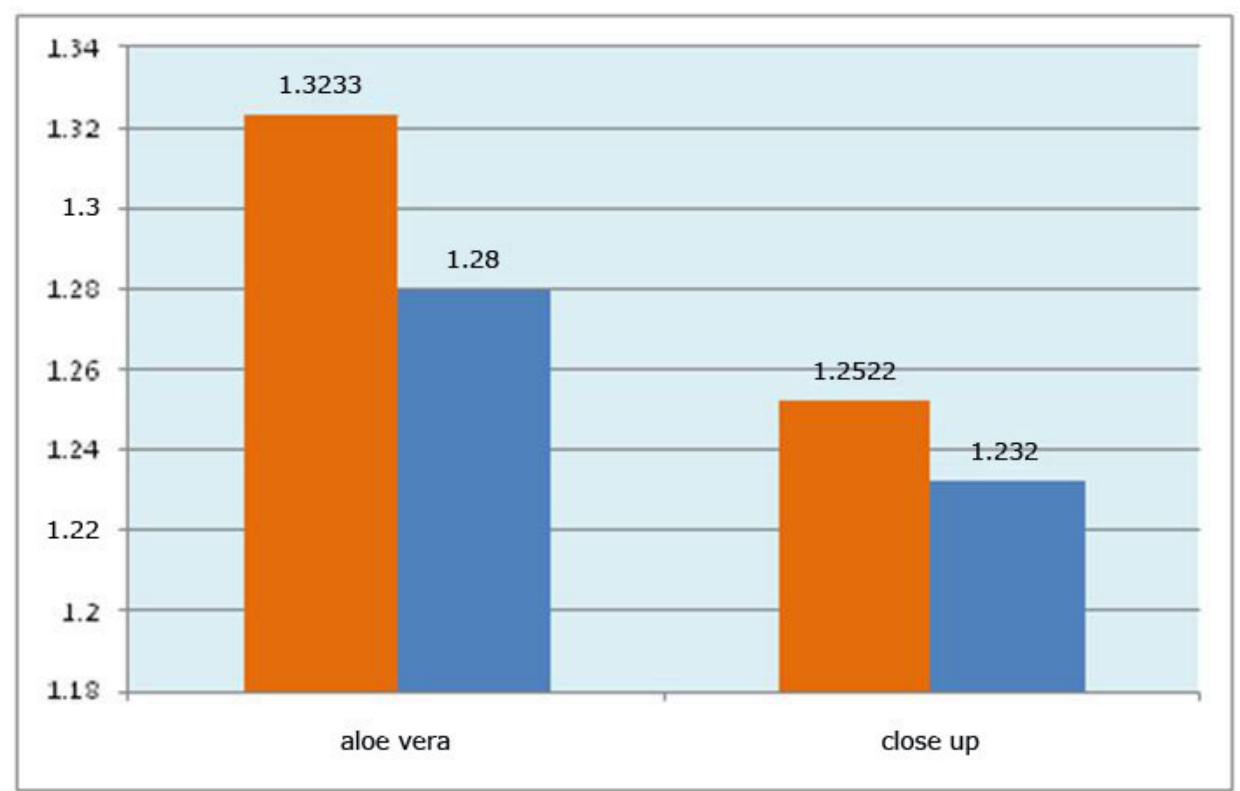

Figure 2: Bar diagram showing the mean gingival index scores for aloe vera and close-up group 
Quigley Hein index

Plaque Scoring Methodology

$0=$ No plaque present.

$1=$ Separate flecks of plaque at the cervical margin.

$2=\mathrm{A}$ thin, continuous band of plaque (up to $1 \mathrm{~mm}$ ) at the cervical margin

$3=\mathrm{A}$ band of plaque wider than $1 \mathrm{~mm}$ but covering less than one-third of the surface.

$4=$ Plaque covering at least one-third but less than twothirds of the surface.

5 = Plaque covering more than two-thirds of the surface

Each tooth is scored in six areas: 1) mesio-facial, 2) midfacial, 3) disto-facial, 4) mesio-lingual, 5) mid-lingual, 6) distolingual. The maximum score per tooth therefore is 30. All teeth are included except third molars and those teeth with prosthetic crowns or cervical restorations. A Plaque Index score for each subject is calculated by adding all the individual plaque scores (six per tooth), and dividing this sum by the total number of measurements (number of teeth scored multiplied by six).

\section{Discussion}

Aloe vera is a natural product contained in commercial herbal dentifrices marketed for the control of plaque and gingivitis. Despite its free commercial use, this phytotherapeutic agent does not have sufficient data to support its anti-gingivitis and anti-plaque claims. Now a days there has been growing interest in natural products. Even though in- vitro trials and studies in animals may show the antimicrobial properties of several of these products, there is no other way of knowing their real clinical effect without conducting a randomized clinical trial [7].

The aloe vera plant has been known and used for centuries for its health, beauty, medicinal and skin care properties. The name aloe vera derives from Arabic word "Alloeh" meaning shining bitter substance, while "vera" in latin means "true" 2000 years ago, the Greek scientists regarded aloe vera as universal panacea. The Egyptians called Aloe "the plant of immortality" [8].

Aloe vera has moisturizing properties, anti-inflammatory, antibacterial, antifungal, antiviral, wound healing, pain relief, treatment of minor burns, skin abrasions, and irritations, treatment of psoriasis and frostbite [6,9-12].

Antimicrobial efficacy of aloe vera tooth gel with 2 commercially available paste was evaluated by George D (2007) in an vitro study and he concluded that aloe vera tooth gel was equally effective against Candida albicans, Streptococcus mutans, Enterococcus faecalis, Prevotella intermedia, Preptosteptococcus anaerobics [6]. Thus demonstrating enhanced antibacterial effect.

Hanley, et al. reported that aloe vera extract decreases inflammation by $48 \%$ in rat adjuvant- induced arthritic inflammatory model [9]. More recently, the peptidase bradykinase was isolated from aloe and shown to break down the bradykinin, an inflammatory substance that reduce pain [13].

This study had compared the aloe vera and close up tooth paste in their efficiency of plaque removal and improving gingival health.

All the participants were asked to use either aloe vera or close up in a cross over manner for a period of 3 weeks and thereafter instructed to continue their regular oral hygiene routines for the following weeks (washout period) [14].

A crossover study was selected because confounding covariates is reduced because each crossover patient serves as his or her own control and these designs are statistically efficient and so require fewer subjects.

Participants were asked to use the aloe vera and close up for 3 weeks because studies have shown that after 9-21 days without proper oral hygiene, healthy gingiva develops heavy accumulations of plaque and generalized marginal gingivitis [2].

The purpose of this study was to determine the efficacy of aloe vera tooth gel and commercially available tooth gel on patients with gingivitis and the results showed that there was significant reduction in plaque scores with both aloe vera and close up tooth paste which was in agreement with those of Villalobos, et al. who observed a significant reduction in plaque and gingivitis after a 30-day use of mouthrinses containing aloe vera associated with toothbrushing [15]. Also, de Oliveira, et al. found that both dentifrice containing aloe vera and dentifrice containing fluoride resulted in significant reduction of plaque and gingivitis, but no statistical significant difference was observed between them [16]. A recent study Pradeep, et al. compared clinical and microbiologic effects of commercially available dentifrice containing aloe vera and found that toothpaste containing aloe vera showed significant improvement in gingival and plaque index scores as well as microbiologic counts compared with placebo dentifrice and these improvements were comparable to those achieved with toothpaste containing triclosan [17].

In present study the percent difference for gingival index with both the tooth paste was not significant at the end of trial which was in disagreement with studies conducted by Villalobos, et al. de Oliveira, et al. and Pradeep, et al. [15-17].

Thus, it can be hypothesized that aloe vera tooth gel can be useful in the control and treatment of gingivitis and reduction of plaque by virtue of its properties.

\section{Conclusion}

The use of natural herbal preparations in oral health care continues to be popular, and aloe vera dentifrice may be a useful addition. Within limits of study, it may be concluded that aloe vera helps in reduction of plaque and gingivitis but did not show any additional 
effects compared to control dentifrice. Additional long-term prospective studies are needed to confirm the results achieved in this study with larger sample size.

\section{References}

1. Carranza FA, Newman MG (2006) Clinical Periodontology ( $10^{\text {th }}$ Edn) W B Saunders Co, USA.

2. Lindhe J, Karring T, Lang NP (2012) Clinical Periodontology and implant dentistry ( $5^{\text {th }}$ edn) Wiley-Blackwell.

3. SP Mangaiyarkarasi, T Manigandan, M Elumalai, Priyanka K Cholan, Roopam Pal Kaur (2015) Benefits of Aloe vera in dentistry. J Pharm Bioallied Sci 7: 55-9.

4. Grindlay D, Reynolds T (1986) The Aloe Vera Phenomenon: A Review of The Properties And Modern Uses Of Theleaf Parenchyma Gel. J Ethnopharmacol 16: 117-51.

5. Bhat G, Kudva P, Dodwad V (2011) Aloe Vera: Nature's Soothing Healer to Periodontal Disease. J Indian Soc Periodontol 15: 205-9.

6. George D, Bhat SS, Antony B (2009) Comparative evalution of antimicrobial efficacy of aloe vera tooth gel and two popular tooth pastes: an in vivo study. Gen dent 57: 238-41.

7. Ozaki F, Pannuti CM, Imbronito AV, Pessotti W, Saraiva L,et al. (2006) Efficacy Of Herbal Tooth Paste On Patients With Established Gingivitis- A Randomized Controlled Trial. Braz Oral Res 20: 172-7.

8. Surjushe A, Vasani R, Saple DG (2008) Aloe Vera: A Short Review. 53: 163-6.

9. Hanley DC, Solomon WA, Saffran B, Davis RH (1982) The evaluation of natural substances in the treatment of adjuvant arthritis. J Am Podiatry Assoc 72: 27584.

10. Rosca- Casian O, Parvu M, Vlase L, Tames M (2007) Antifungal activity of aloe vera leaves. Fitoterapia 78: 219-22.

11. Saoo K, Miki H, Ohmori M, Winters WD (1990) Antiviral activity of aloe extracts against cytomegalovirus. Phytother Res 10: 348-50.

12. Wynn RL (2005) Aloe vera gel: update for dentistry. Gen dent 23: 6-9.

13. Davis RH, Parker WL, Samson RT, Murdoch DP (1991) Isolation of a stimulatory system in an aloe extract. J Am Podiatr Med Assoc 81: 473-8.

14. Bhambal A, Kothari S, Saxena S, Jain M (2011) Comparative effect of Neemstick and Toothbrush on Plaque Removal and Gingival Health- a Clinical Trial. J Of Advanced Oral Research 2: 51-4.

15. Villalobos OJ, Salazar CR, Sanchez Gr (2001) Effect of a Mouthwash made of Aloe Vera on plaque and gingival inflammation (In Spanish) Acta Odontol Venez 39: 16-24.

16. De Oliveira SM, Torres TC, Pereira SL, Mota OM, Carlos MX (2008) Effect of a Dentifrice Containing Aloe Vera On plaque and Gingivitis Control. A DoubleBlind Clinical study In Humans. J Appl Oral Sci 16: 293-6.

17. Pradeep AR, Agarwal E, Naik SB (2012) Clinical and Microbiologic Effects of Commercially Available Dentifrice Containing Aloe Vera: A Randomized Controlled Clinical Trial. J Periodontol 83: 797-804. 